\title{
Traceability validation of a high speed short-pulse testing method used in LED production
}

\author{
Elena Revtova*, Edgar Moreno Vuelban, Dongsheng Zhao, Jacques Brenkman, and Henk Ulden \\ VSL BV, Dutch Metrology Institute, Thijsseweg 11, 2629JA Delft, Netherlands
}

Received: 22 August 2017 / Accepted: 6 November 2017

\begin{abstract}
Industrial processes of LED (light-emitting diode) production include LED light output performance testing. Most of them are monitored and controlled by optically, electrically and thermally measuring LEDs by high speed short-pulse measurement methods. However, these are not standardized and a lot of information is proprietary that it is impossible for third parties, such NMIs, to trace and validate. It is known, that these techniques have traceability issue and metrological inadequacies. Often due to these, the claimed performance specifications of LEDs are overstated, which consequently results to manufacturers experiencing customers' dissatisfaction and a large percentage of failures in daily use of LEDs. In this research a traceable setup is developed to validate one of the high speed testing techniques, investigate inadequacies and work out the traceability issues. A well-characterised short square pulse of $25 \mathrm{~ms}$ is applied to chip-on-board (CoB) LED modules to investigate the light output and colour content. We conclude that the short-pulse method is very efficient in case a well-defined electrical current pulse is applied and the stabilization time of the device is "a priori" accurately determined. No colour shift is observed. The largest contributors to the measurement uncertainty include badly-defined current pulse and inaccurate calibration factor.
\end{abstract}

Keywords: high speed short-pulse method / LED packages and modules / CoB LED module / SSL product / colour shift / electrical pulse characteristics / direct current (DC) / traceability / temperature dependency

\section{Introduction}

A measurement gap is identified between the way LED manufacturers test single (high power) LEDs and LED packages/modules (including remote-phosphor) during production and the way lighting calibration and testing laboratories calibrate/test SSL products.

In industry, the so-called single-pulse method $[1,2]$ limits LED power by powering the LED with a single current pulse or series of multiple pulses, usually in the length of 50-300 ms. Such pulses are short enough to limit LED package heating but not short enough to limit junction heating [2]. In some cases, LED manufacturers use pulse-mode operation with a very short pulse, typically 10 or $20 \mathrm{~ms}$, which will not heat up the LED [3].

The main objectives of this research are threefold: (1) to validate the high speed short-pulse testing method of LEDs for its applicability by pointing out any inadequacies (e.g. inaccurate characteristics of driving pulse, synchronization problem between driving and measuring equipment, and discrepant and fluctuating temperature setting), (2) to provide traceability to the measurands through the

\footnotetext{
* Corresponding author: rewtowa@gmail.com
}

determination of traceable conversion factors to the values, and (3) to propose a new method. These measurands are obtained in DC (direct current) operating conditions, and including the presence of time dependency.

The full characterization of the measurement setup (replicating the industrial setup) and the validation of the short-pulse testing method, in general, should lead to an improvement of the way how manufacturers perform the testing and sorting of their LED products and to the reduction of errors in LED performance evaluation testing.

\section{Calibration laboratory versus industrial testing}

The main measurand during the final testing of a LED source at manufacturer (single LED or LED package/ module) is the total luminous flux $\Phi_{v}$ in [lm].

In the calibration laboratory conditions (e.g. NMI (National Metrology Institute)), the total luminous flux of an SSL product (a lighting product based on a LED source), is normally determined using an integrating sphere facility corrected for spectral mismatch and non-homogeneity. Traceability is provided through the use of a 
standard lamp with the known luminous flux in the socalled relative method.

$$
\Phi_{v}^{S S L}=\frac{\Phi_{v}^{s} \cdot I_{m}}{I_{m}^{s}} \cdot k,
$$

where $\Phi_{v}^{S S L}$ is the determined total luminous flux of the SSL product, $\Phi_{v}^{s}$ is the total luminous flux of the standard lamp, $I_{m}$ and $I_{m}^{s}$ are the measured electrical currents from the optical detector and standard lamp respectively, and $k$ is the total correction factor.

In the laboratory setup, the SSL product is normally driven under DC condition and enough time is taken to reach a stable light output according to a measurement standard (the most recent standard is the EN 13032-4 [4]). For industrial testing, this DC operating method used mostly in calibration laboratories is not applicable due to time limitations, and also due to the fact that the light output of LEDs very strongly depends on temperature. Therefore, a quicker method is adopted by industry to avoid high rise of temperature and to reduce the stabilisation time of LEDs. This method is based on the use of a single or multiple short pulse(s) to drive the LEDs.

\section{DC versus pulsed operation of LEDs}

This section is dedicated to understanding of the differences between a DC-driven and pulsed-driven LED source (further referred to as Device Under Test (DUT)). A simplified theory, including the assumptions made during data analysis, is presented to describe the behaviour of the DC-driven and pulsed-driven DUTs.

Let us start with the total radiant flux or power $\Phi_{e}$ of a DUT in [W] which may be estimated according to [5] by the following equation:

$$
\Phi_{e}=\frac{\partial Q_{e}}{\partial t}=\int P(\lambda) \cdot \mathrm{d} \lambda
$$

where $P(\lambda)$ is the absolute spectral radiant power distribution function of the DUT, with unit in $[\mathrm{W} / \mathrm{nm}]$.

In "lighting" metrology, however, the total luminous flux $\Phi_{v}$ is most valuable quantity than the total radiant flux as this is the part of useful radiation sensed by human eye and is the quantity which determines the efficiency of a light source under test. The total luminous flux may be estimated using the modified equation (2) following [6]:

$$
\Phi_{v}=K_{m} \cdot \int_{\lambda_{\min }}^{\lambda_{\max }} P(\lambda) \cdot V(\lambda) \cdot d \lambda,
$$

where $K_{m}$ is the maximum spectral luminous efficacy of radiation for photonic vision which is $683[\mathrm{~lm} / \mathrm{W}]$, $\lambda_{\min }=380 \mathrm{~nm}, \lambda_{\max }=780 \mathrm{~nm}$ and $V(\lambda)$ is the relative function which is called the spectral luminous efficiency for photonic vision $[5,7]$.

To determine directly the total luminous flux, based on the above equation, is nontrivial since this requires accurate knowledge of the absolute spectral power distribution $P(\lambda)$ of the DUT, which is not an easy task. Therefore, in industry and testing laboratories another relative method is normally used, which is known as integrating sphere method already described in Section 2.

In this research, we used the absolute calibration method. The starting point in this study is then the equation, though the function $P(\lambda)$ is only known relatively from the measurements of the DUT with a spectroradiometric system in the range from 380 to $780 \mathrm{~nm}$. Thus we may rewrite the equation in terms of relative power function $P^{r e l}(\lambda)$ :

$$
\Phi_{\nu}=K_{m} \cdot x \cdot \int_{\lambda_{\min }}^{\lambda_{\max }} P^{r e l}(\lambda) \cdot V(\lambda) \cdot d \lambda
$$

with $x$ being then a scaling factor (with unit in [W]) between the relative and absolute power of the DUT.

In this research, we focused on the determination of the absolute power distribution $P(\lambda)$ of the DC and short-pulse driven sources for the purpose of traceability validation. The main emphasis was on determination of the scaling factor $x$ as accurate as possible.

\subsection{DC driving operation}

In this study, the traceability is coming from the developed fast Optical Detector System (ODS) with the filter wheel used for detecting partial signal, in terms of electrical current $I_{i}^{p}$ for each filter $i$. The ODS was calibrated on the Absolute Cryogenic Radiometer (ACR) facility at VSL (NMI of the Netherlands) in terms of its spectral responsivity per each filter $I, R_{i}^{d}(\lambda)$ in $[\mathrm{A} / \mathrm{W}]$. Additionally, the uncertainties of the spectral responsivity functions have been estimated.

In general, the spectral responsivity function, $R_{i}^{d}(\lambda)$ (unit in $[\mathrm{A} / \mathrm{W}]$ ), of a filter $i$ of the ODS can be described by the following expression:

$$
R_{i}^{d}(\lambda)=\frac{I_{i}(\lambda)}{P_{S}(\lambda)}
$$

where $I_{i}(\lambda)$ is the corresponding electrical current (unit [A]) of the light output, for each particular wavelength $\lambda$ (unit in $[\mathrm{nm}])$, and $P_{s}(\lambda)$ is the spectral power distribution function of the standard source (unit in [W]). Normally an incandescent "flat spectrum" source is used for calibration and currents have normally levels in the $\mu \mathrm{A}$.

Knowing the spectral responsivity functions $R_{i}^{d}(\lambda)$ and the total signal measured by the filter $i$ of the ODS, $I_{i}^{p}$, the partial power $P_{i}^{p}$ of the SSL product under test obtained by the filter $i$ may be determined using the following equation:

$$
P_{i}^{p}=\frac{I_{i}^{p}}{R_{i}^{d}\left(\lambda_{i}\right)}
$$

with $\lambda_{i}$ being the peak wavelength of the filter $i$ provided by the manufacturer.

In the ideal situation, function $P_{i}^{p}$ would represent the power of the DUT at the single wavelength $\lambda_{i}$. In this study, the spectral responsivity functions of the ODS differ from 
the Dirac delta function having the centroid wavelengths at $\lambda=\tilde{\lambda}_{i}$ and the form is defined by the spectral responsivity function $R_{i}^{d}(\lambda)$. The centroid wavelength $\tilde{\lambda}_{i}$ may differ from the one given in the specification of the manufacturer, $\lambda_{i}$, by the order of $0.5-1.0 \mathrm{~nm}$, and this should be taken into account in the data analysis and uncertainty estimation.

Knowing the spectral responsivity functions $R_{i}^{d}(\lambda)$ of the ODS for each filter $i$, we may calculate the centroid wavelength $\tilde{\lambda}_{i}$ using the following expression ([5]):

$$
\tilde{\lambda}_{i}=\frac{\int_{\lambda_{\min }}^{\lambda_{\max }} \lambda \cdot R_{i}^{d}(\lambda) \cdot d \lambda}{\int_{\lambda_{\min }}^{\lambda_{\max }} R_{i}^{d}(\lambda) \cdot d \lambda},
$$

which may be substituted by the numerical expression:

$$
\tilde{\lambda}_{i}=\frac{\sum_{\lambda_{\min }}^{\lambda_{\max }} \lambda \cdot R_{i}^{d}(\lambda) \cdot \Delta \lambda}{\sum_{\lambda_{\min }}^{\lambda_{\max }} R_{i}^{d}(\lambda) \cdot \Delta \lambda}
$$

Additionally, using the relative spectral distribution function of the DUT, $P^{r e l}(\lambda)$, we may reconstruct the wavelength dependent currents $I_{i}(\lambda)$ from the integral $I_{i}^{p}$ to obtain the absolute power $P_{i}$, of the source at the centroid wavelength $\tilde{\lambda}_{i}$, using the series of following equations:

$$
I_{i}^{r e l}(\lambda)=\frac{I_{i}(\lambda)}{x_{i}}=P^{r e l}(\lambda) \cdot R_{i}^{d}(\lambda),
$$

with $I_{i}^{r e l}(\lambda)$ being the relative current of each filter per wavelength which we will scale further by a factor $x_{i}$ to obtain the absolute spectral current $I_{i}(\lambda)$. The scaling factor $x_{i}$ is determined by the next three equations (10)(12) and the absolute spectrally dependent current by equation (13) using numerical integration in the measurement region of wavelengths between $\lambda_{\min }$ and $\lambda_{\text {max }}$ :

$$
\begin{gathered}
I_{i}^{p}=\sum_{\lambda_{\min }}^{\lambda_{\max }} I_{i}(\lambda) \cdot \Delta \lambda=\sum_{\lambda_{\min }}^{\lambda_{\max }} I_{i}^{r e l}(\lambda) \cdot x_{i} \cdot \Delta \lambda, \\
I_{i}^{p}=x_{i} \cdot \sum_{\lambda_{\min }}^{\lambda_{\max }} P^{r e l}(\lambda) \cdot R_{i}^{d}(\lambda) \cdot \Delta \lambda, \\
x_{i}=\frac{I_{i}^{p}}{\sum_{\lambda_{\min }}^{\lambda_{\max }} P^{r e l}(\lambda) \cdot R_{i}^{d}(\lambda) \cdot \Delta \lambda}, \\
I_{i}(\lambda)=x_{i} \cdot I_{i}^{r e l}(\lambda)=\frac{P^{r e l}(\lambda) \cdot R_{i}^{d}(\lambda) \cdot I_{i}^{p}}{\sum_{\lambda_{\min }}^{\lambda_{\max }} P^{r e l}(\lambda) \cdot R_{i}^{d}(\lambda) \cdot \Delta \lambda} .
\end{gathered}
$$

To determine the absolute power of the source at the centroid wavelengths $\tilde{\lambda}_{i}$ for all the filters, the following equation was used:

$$
\frac{P_{i}=I_{i}\left(\tilde{\lambda}_{i}\right)}{R_{i}^{d}\left(\tilde{\lambda}_{i}\right)=x_{i} \cdot P_{i}^{r e l}\left(\tilde{\lambda}_{i}\right)}
$$

\subsection{Pulsed driven operation}

The derivation performed above is valid when a constant DC current is applied to drive a temperature-stabilized DUT and time dependence is not an issue. In industry, during the performance testing of LED sources a short pulse of width of $25-50 \mathrm{~ms}$ is normally applied to feed it instead of a DC to avoid obvious temperature rise. In such a case, the derivations become more complex. An extra dependence on time is appearing and time-resolved measurements are required to understand what is happening with the light output of the source sensed by the optical system.

Basically, a square short pulse of width $t_{w}$, when applied to the DUT, causes the source to respond correspondingly to the temporal profile of the pulse but not necessarily equal to pulse-width $t_{w}$. In industry, during light output performance testing of LED sources the time integral of the total luminous output of the source driven by the pulse of width $t_{w}$ is normally measured with an integrating sphere setup. Let us name it $I^{\text {pulse }}$. The resulting total luminous flux $\Phi_{v}^{\text {pulse }}$ of the short-pulse driven DUT differs from the total luminous flux $\Phi_{v}$ of the DC-driven DUT with a factor $s$. The main difficulty is then to estimate this factor as accurate as possible. This factor $s$ highly depends on the technology, processing complexities, device specifications, and characteristics of each single DUT.

In this research we obtained the spectrally-resolved (per filter $i$ ) and time-resolved partial light output of the DUT, $I_{i}^{p t}(t)$, by the ODS developed at VSL. This timeresolved partial light output for each filter of ODS, the $I_{i}^{p t}(t)$, is known as a convolution of two functions: (1) the time-resolved current obtained by the ideal optical system when filtered by filter $i, I_{i}^{p}(t)$ and (2) the response time function of the developed ODS, $R^{t}(t)$.

In this study, we made an assumption that the response time of the ODS is uniform for all the filters $i$ and is simply equal to $R^{t}(t)$. The time-resolved partial light output for each filter of ODS may be written then by the following equation:

$$
I_{i}^{p t}(t)=I_{i}^{p}(t) R^{t}(t)=\int_{-\infty}^{\infty} I_{i}^{p}(t-\tau) \cdot R^{t}(\tau) \cdot d \tau .
$$

As we worked in finite domain, a numerical approximation may be used and the equation (15) may be substituted by a summation:

$$
I_{i}^{p t}(t)=\sum_{0}^{t} I_{i}^{p}(t-\tau) \cdot R^{t}(\tau) \cdot \Delta \tau
$$

Furthermore, we used $I_{i}^{p t}(t)$ to estimate the time-resolved total power $P_{i}^{\text {pulse }}(t)$ of the light output from the shortpulse driven DUTs obtained by the filter $i$ in the same way as it is shown in equation (6) by the following expression:

$$
\left.P_{i}^{p u l s e}(t)=\frac{I_{i}^{p t}(t)}{R_{i}^{d}\left(\tilde{\lambda}_{i}\right.}\right)
$$

with $R_{i}^{d}\left(\tilde{\lambda}_{i}\right)$ being the spectral responsivity of the ODS with filter $i$ at the calculated centroid wavelength $\tilde{\lambda}_{i}$. 
For evaluation purposes, we have to obtain $P_{i}^{\text {pulse }}$ for each filter for the short-pulse driven DUT, independent of time, the same as, $P_{i}$ for the DC-driven DUT.

To obtain the time integrated absolute power of the source $P_{i}^{\text {pulse }}$ and $P_{i}$ at the centroid wavelength of the filter $i$, we used two approaches: 1) maximum signal for shortpulse driven DUT and 2) an iterative approach based on the estimation of the median within the DC (the long pulse of $2.5 \mathrm{~s}$ in this study). For the iterative approach, the standard deviations (further STD) of the medians from different iterations were compared with the convergence criterion $C_{m}$. The value of $C_{m}$ is based on the resolution limit of the electrical measurement instrumentation. In our case, the lowest resolution of the measured current from ODS is $4 \mu \mathrm{A}$, which is around $2 \%$. The iterative method may be given by a flowchart given below:

Equation (8) was used to calculate the centroid wavelength $\tilde{\lambda}_{i}$. Furthermore, the series of equations (9)(14) and (17) were applied to calculate the scaling factors $x_{i}$ and to determine the absolute power of the DC-driven DUT, $P_{i}$ and short-pulse driven DUT, $P_{i}^{\text {pulse }}$, at the calculated centroid wavelengths.

In general, it is assumed that in case no spectral shift is observed and no errors are present, then all the $x_{i}$ shall be equal to $x$. Due to errors and approximations used, but also different physical behaviour, a difference between the scaling factors $x_{i}$ may show: 1 ) a measurement error and 2) a spectral shift. Therefore, an average $x$ shall be obtained and its error shall be estimated.

\section{Measurement setup, conditions and traceability chain}

In our laboratory, a traceable setup which is capable of driving LEDs and LED modules under DC and short pulses, has been realized. The setup is also capable of spectrally-resolved as well as time-resolved measurements of light output of DUTs.

A schematic of the complete measurement setup is shown in Figure 1. The setup consists of three functional blocks related to 1) electrical driving equipment, 2) optical measuring equipment and 3) electrical measuring equipment. A PC has been used to control the experiments and collect the data. A Labview software has been developed by VSL for the data collection from the measuring equipment.

A high voltage pulse shaper HV1000 was powered by a DC voltage source (Heinzinger). A predefined $5 \mathrm{~V}$ pulse was generated by the SRS pulse generator (DG645) and was used as input to trigger the HV1000 pulse shaper. The high voltage pulse output from the HV1000 was then used to drive the DUTs. A fast rise time module (SRD1) was additionally used for the SRS DG645 pulse generator to improve the pulse shape.

For the optical measurements, the ODS has been used. The ODS consists of a filter wheel from Thorlabs (type FW102C) filled with calibrated narrow-band spectral filters and an in-house developed Si-based trap detector for high speed measurements. The temperature of the ODS filter wheel is controlled at the level of $t=30 \pm 0.5^{\circ} \mathrm{C}$.

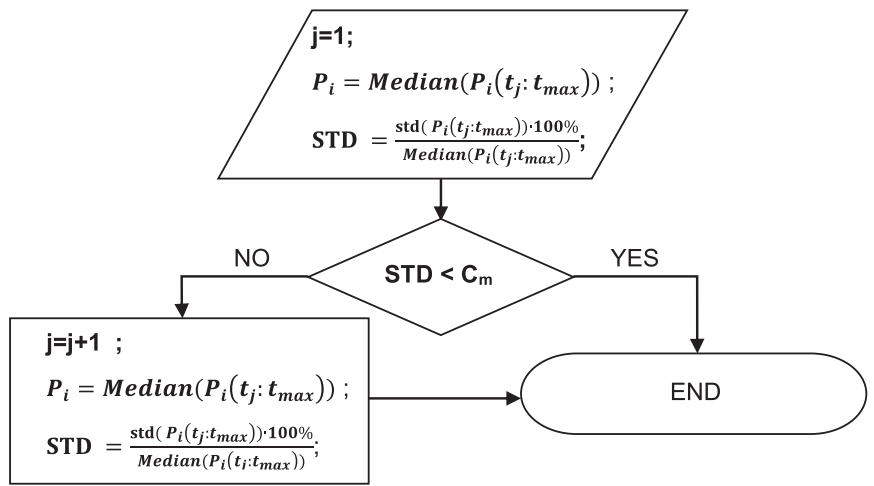

Fig. 1. The flowchart of the iterative method to obtain the time integrated absolute power of the DUT.

Six commercially available laser-band filters (Semrock) have been purchased and pre-tested on their spectral transmission. Thereafter, four filters have been selected and the spectral shape (improved suppression of the higher wavelength area) of two filters was improved by combining them with Thorlabs short-pass filters (refer to Tab. 1 for specifications).

A precise power oscilloscope (Yokogawa PX8000) calibrated at VSL (Electrical Group) has been used for the electrical measurements of the driving equipment and the light output from ODS.

Optical and electrical measurements have been performed in the laboratory at controlled ambient conditions with the temperature of $T=23.0 \pm 0.5^{\circ} \mathrm{C}$ and humidity of $35 \pm 5 \%$.

Traceability chart is given in Figure 2. ODS is traceable to the spectral responsivity of the Absolute Cryogenic Radiometer (ACR).

\section{Characterisation and calibration of equipment}

\subsection{Characterization of driving electrical equipment}

The electrical driving signal of the setup has been characterized using an oscilloscope. Voltage pulse of $5 \mathrm{~V}$ coming directly from the trigger DG465 has been measured and compared with the pulsed signal of $30 \mathrm{~V}$ shaped by VSL-modified pulse shaper (HV1000).

After modification of the HV1000 to give a high voltage pulse shaped from $5 \mathrm{~V}$ trigger, a $50 \mathrm{~Hz}$ sinusoidal ripple of about $100 \mathrm{mV}$ is observed on the unloaded curve. The noise of the load curve (SSL device) may be estimated to be not larger than $50 \mathrm{mV}$ at $20 \mathrm{~V}$ and $250 \mathrm{mV}$ at $30 \mathrm{~V}$. The results obtained for the pulse characteristics are shown in Table 2.

\subsection{Calibration of the ODS}

Traceable spectrally-resolved as well as time-resolved calibrations of ODS were performed to investigate the main contributors to the uncertainty budget. 
Table 1. Specification of the filters used in the filter wheel of the ODS.

\begin{tabular}{|c|c|c|c|c|}
\hline NN Filter, ODS & Filter type and ID & $\begin{array}{l}\text { Wavelength, } \\
\text { specification, } \\
\mathrm{nm}\end{array}$ & $\begin{array}{l}\text { FWHM bandwidth, } \\
\text { typical, } \\
\text { nm }\end{array}$ & $\begin{array}{l}\text { Transmission, } \\
\text { specification, } \\
\%\end{array}$ \\
\hline F633 & Thorlabs Narrow Band FLH633-5 & 633 & 5 & $>95 \%$ \\
\hline F532 & Thorlabs Narrow Band FLH532-4 & 532 & 4 & $>92 \%$ \\
\hline F488 & $\begin{array}{l}\text { Combination of Thorlabs Short pass FESH0550 } \\
\text { and Laser Band Semrock LL01-488-25-D }\end{array}$ & 488 & 1.6 & $>95 \%$ \\
\hline F458 & $\begin{array}{l}\text { Combination of Thorlabs Short pass FESH0500 } \\
\text { and Laser Band Semrock LL01-458-25-D }\end{array}$ & 458 & 1.7 & $>95 \%$ \\
\hline
\end{tabular}

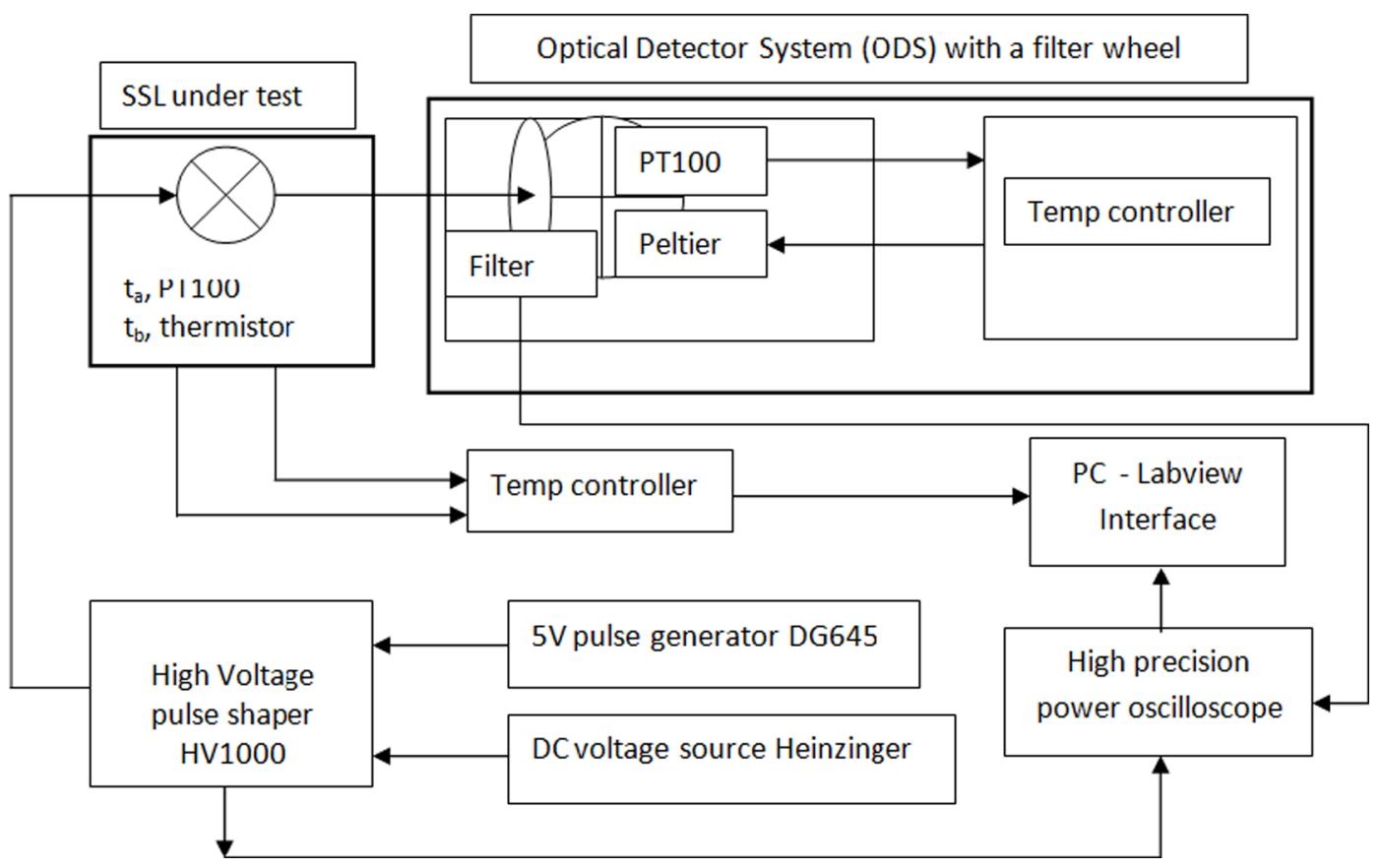

Fig. 2. Schematic of the traceable test setup developed at VSL.

Table 2. Characteristics of the triggering and driving equipment.

\begin{tabular}{llllll}
\hline Signal & Pulse-width & Rise time & Overshoot rise & Fall time & Overshoot fall \\
\hline $5 \mathrm{~V}$ trigger & $25 \mathrm{~ms}$ & $100 \mathrm{ps}$ & $0.0 \%$ & $3 \mu \mathrm{s}$ & $0.0 \%$ \\
$40 \mathrm{~V}$ output pulse & $25 \mathrm{~ms}$ & $6 \mu \mathrm{s}$ & $3 \%$ & $10 \mu \mathrm{s}$ & $0.0 \%$ \\
\hline
\end{tabular}

The ODS has been calibrated on the ACR facility at VSL multiple times. This facility is used to calibrate detectors (sensors) on their absolute spectral responsivity in $\mathrm{A} / \mathrm{W}$. The resulting spectral responsivity functions of each filter are shown in Figure 3.

Two filters F458 and F488 have lower spectral responsivity due to higher suppression of the signal by the combination of two filters (Semrock laser band and Thorlabs short pass).

As to the response time of the ODS, it has been investigated within the EMRP (European Metrology Research Programme) ENG05 project "Metrology for Solid
State Lighting" [8]. The device shows nearly perfect linear behaviour down to $10 \mu \mathrm{s}$ which is shown in Figure 4. The response time of the system is primarily limited by the bandwidth of the transimpedance amplifier.

In the case of $25 \mathrm{~ms}$ pulse, which was used to drive the DUTs in this study, the time dependency of the ODS system is considered to be negligible and the response time is assumed to be a linear function with the average response time $\bar{R}^{t}=1$. Using this assumption, the equation (15) can be simplified to:

$$
I_{i}^{p t}(t)=I_{i}^{p}(t)
$$



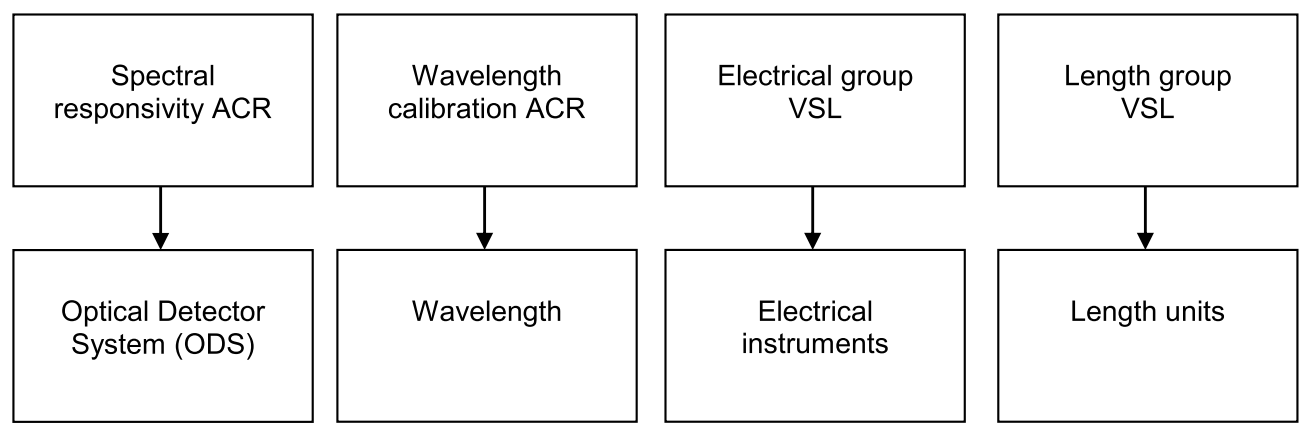

Fig. 3. Traceability chart. ACR stands for Absolute Cryogenic Radiometer.

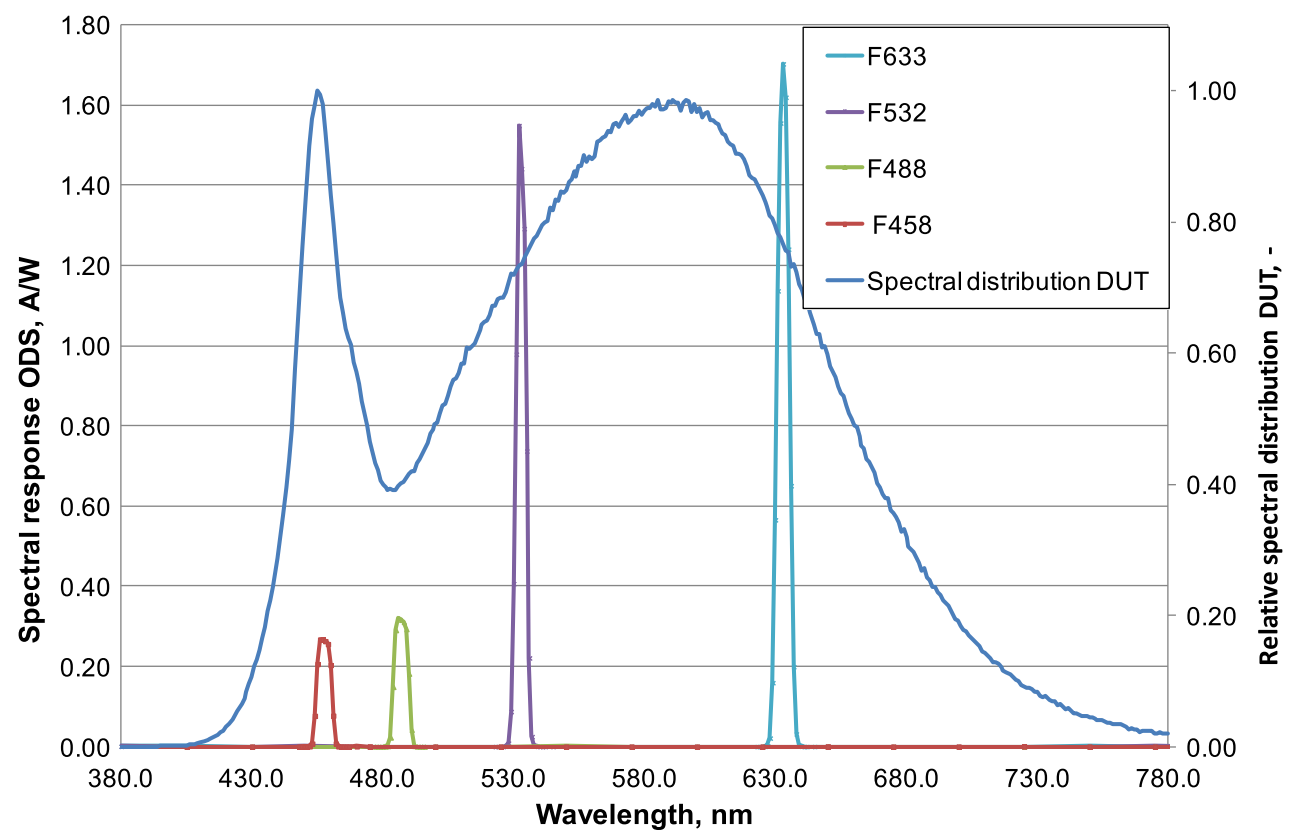

Fig. 4. Absolute Spectral Responsivity of the ODS filters F633, F532, F488 and F458 (A/W), and the relative spectral distribution power of a DUT normalized to maximum (secondary axis).

\subsection{Luminous flux calibration of the DUTs}

To characterise the DUTs and to monitor their ageing, the pre-burned DUT has been calibrated on integrating sphere facility at VSL before and after the experiments on its total luminous flux and spectral characteristics. Measurement results of the characterisation are summarised in Table 3.

\section{Measurement procedure and data analysis}

Every DUT has been driven by DC and short pulse $(25 \mathrm{~ms})$. In this research, a DC condition has been set by applying a single long pulse of $2.5 \mathrm{~s}$ duration. The timeresolved measurement of the spectrally filtered signal was performed using the developed ODS connected to the precise power oscilloscope. The ambient temperature and the surface temperature of the DUTs were measured every second. The input electrical time-
Table 3. Resulting characteristics of the pre-burned DUT (ID VSL085) before and after the experiments.

\begin{tabular}{llll}
\hline $\begin{array}{l}\text { Measurand, } \\
\text { unit }\end{array}$ & $\begin{array}{l}\text { Before testing } \\
\text { but after 100h } \\
\text { pre-burning }\end{array}$ & $\begin{array}{l}\text { After } \\
\text { testing }\end{array}$ & Difference \\
\hline Luminous Flux, lm & 3061 & 3028 & 33 \\
CCT, K & 3985 & 4023 & 38 \\
$x$ & 0.3840 & 0.3821 & -0.0019 \\
$y$ & 0.3876 & 0.3860 & -0.0016 \\
\hline
\end{tabular}

resolved driving forward voltage and the power consumption were measured with the sampling time interval of $1 \mu \mathrm{s}$. 
Table 4. Parameters of the pulsed and DC experiments.

\begin{tabular}{lll}
\hline Parameter & Pulsed mode & DC mode \\
\hline Driving voltage CoB, V & 41 & 41 \\
& $100(\mathrm{~F} 488$, & $100(\mathrm{~F} 488$, \\
Amplifier gain setting & $\mathrm{F} 458)$ & $\mathrm{F} 458)$ \\
of the ODS, k 2 & $10(\mathrm{~F} 633$, & $10(\mathrm{~F} 633$, \\
& $\mathrm{F} 532)$ & $\mathrm{F} 532)$ \\
Trigger rate, $\mathrm{Hz}$ & 1 & 0.025 \\
Pulse-width, ms & 25 & 2500 \\
Set point ambient & 25.1 & 25.1 \\
temperature, ${ }^{\circ} \mathrm{C}$ & & \\
Tolerance temperature, ${ }^{\circ} \mathrm{C}$ & 0.5 & 0.5 \\
\hline
\end{tabular}

Table 4 shows the settings of the driving and measuring equipment during the experiments. The driving voltage was kept close to the recommended value by the manufacturer at the level of $41 \mathrm{~V}$. A transimpedance amplifier system developed at VSL has been used to amplify the ODS signal with the amplifier gain setting of 10 or $100 \mathrm{k} \Omega$ depending on the filter of the ODS.

The list of measured parameters is given below:

- spectrally filtered time-resolved optical signal obtained by ODS, $I_{i}^{p t}(t)$;

- electrical trigger voltage, $U_{i}^{f}$;

- measured electrical power $\left(P^{m}\right)$, current $\left(I^{m}\right)$, voltage $\left(V^{m}\right)$;

- DUT surface temperature, $T_{s}$;

- ambient temperature, $T_{a}$.

All the parameters from the list given above were measured and transferred to the PC LabView program developed at VSL.

\section{Testing results}

\subsection{Time-resolved measurements}

Resulting light output of the two DUTs (CoB modules of type OSRAM SOLERIQ E 30 Series White 4000K LED, $120^{\circ}$ ) obtained by the ODS is given in Figures 5-8. Figures 5 and 6 show the time-resolved current of the DCdriven DUT measured by each ODS spectral filter and Figures 7 and 8, driven by the short pulse-width of $25 \mathrm{~ms}$. The driving time-resolved voltage is also shown in all the figures for reference.

The shape of the time-resolved light output is very close to the one previously mentioned in [9]. The shape confirms the response of the LED output on junction temperature rise. In [9] it is pointed that for the pulsewidth between $50 \mathrm{~ms}$ and $300 \mathrm{~ms}$ "often the LED's junction temperature increases from 10 to $30^{\circ} \mathrm{C}$ above the case temperature by the end of the pulse". We expected the same behaviour of the DUT in this research for the DC-driving experiments.

In this study, we have not performed time-resolved measurements of the LED temperature. As it has been mentioned above, we only monitored ambient and surface temperatures at $1 \mathrm{~s}$ time interval.
A cut of the short-pulse driven DUT light output is clearly observed in Figures 7 and 8. It is therefore expected that the absolute power of the short-driven DUT shall be lower than that of the DC-driven.

\subsection{Absolute spectral power}

The iterative method described in Section 3.2 has been used to evaluate the time integral light output of the ODS in terms of the electrical current within the DC-driven DUT. For the short-pulse experiments, a maximum value was used as no stability of DUT was reached within the shortpulse. Figure 9 gives the impression of calculations for the DC experiment: (1) the measured current by ODS, (2) its iterative medians and (3) standard deviations used for convergence evaluation.

The centroid wavelengths of the filters calculated using equation (8) and resulting wavelengths are given in Table 5. The calculated centroid wavelengths of the filters differ from the specification of the manufacturer to the values between 0.3 and $0.9 \mathrm{~nm}$.

Following the described method given in Section 3.2, the scaling factors $x_{i}$ and the absolute spectral radiant power for each ODS filter have been calculated for both experiments: (1) DC-driven, $P_{i}$, and (2) short-pulse driven, $P_{i}^{\text {pulse }}$. The resulting scaling factors $x_{i}$ as estimated using these two DUTs are given in Table 6. The STD of the scaling factors $x_{i}$ under the same driving condition (DC or short-pulse) is much larger than expected, which is around $10 \%$ for all the experiments. Ideally, $x_{i}$ is a constant value. It is also quite obvious that the resulting factors $x_{i}$ at $\lambda_{i}=633 \mathrm{~nm}$ are $15-18 \%$ larger than the rest. The difference between the scaling factors for the same filter but for different electrical driving conditions (DC or short-pulse driven) is between $6-11 \%$.

The resulting reconstructed absolute spectral power of the short-pulse driven DUT VSL085 $\left(P_{i}^{\text {pulse }}\right)$ and DCdriven $\left(P_{i}\right)$ are given in Figures 10 and 11 . The scaled spectral distribution function of the DUTs, $P(\lambda)$, are also given in the figures for reference. They have been obtained from the relative power distribution functions of DUTs, $P^{r e l}(\lambda)$, measured by the spectroradiometer in the integrating sphere facility at VSL, by scaling it with the averaged $x$.

In Table 7, the resulting differences between the absolute power values of short-pulse driven and DC-driven DUTs are given per filter of ODS. The same resulting differences as for the scaling factor $x_{i}$ have been observed: $6-11 \%$ between DCdriven and short-pulse driven DUTs. The same differences have the same source as radiant power values what is expected when analysing the equations in Section 3.

\subsection{Ambient temperature dependence of the DUT}

To estimate the ambient temperature dependence of the light output of the DUTs and the uncertainty contribution due to temperature change, an extra experiment was performed for short-pulse driven DUTs. Both devices under test have been tested at four different ambient temperatures $T_{a}$ of $25,30,35$ and $40^{\circ} \mathrm{C}$. An increase of the 


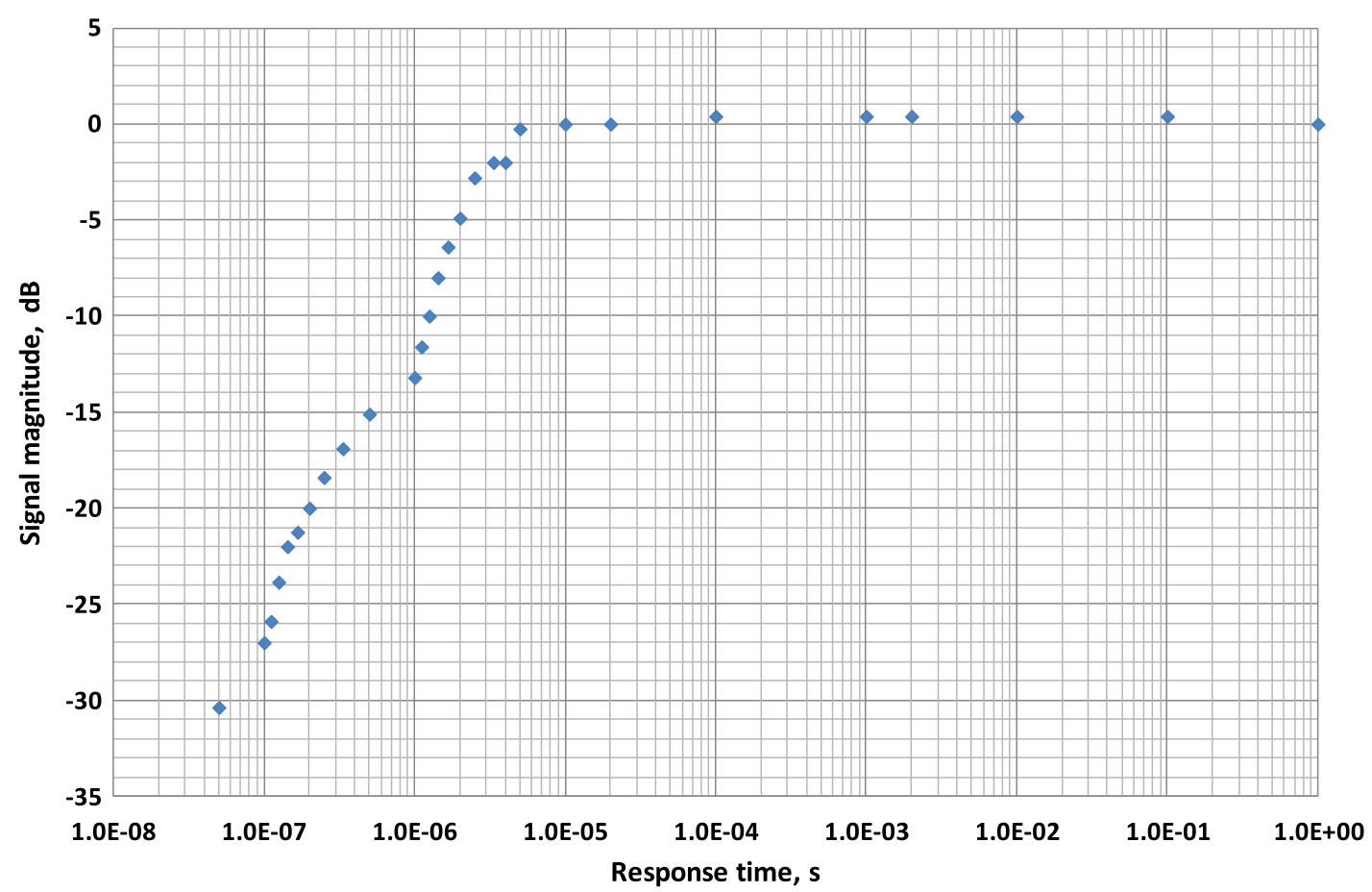

Fig. 5. Response time of the ODS as it was characterized in [8].

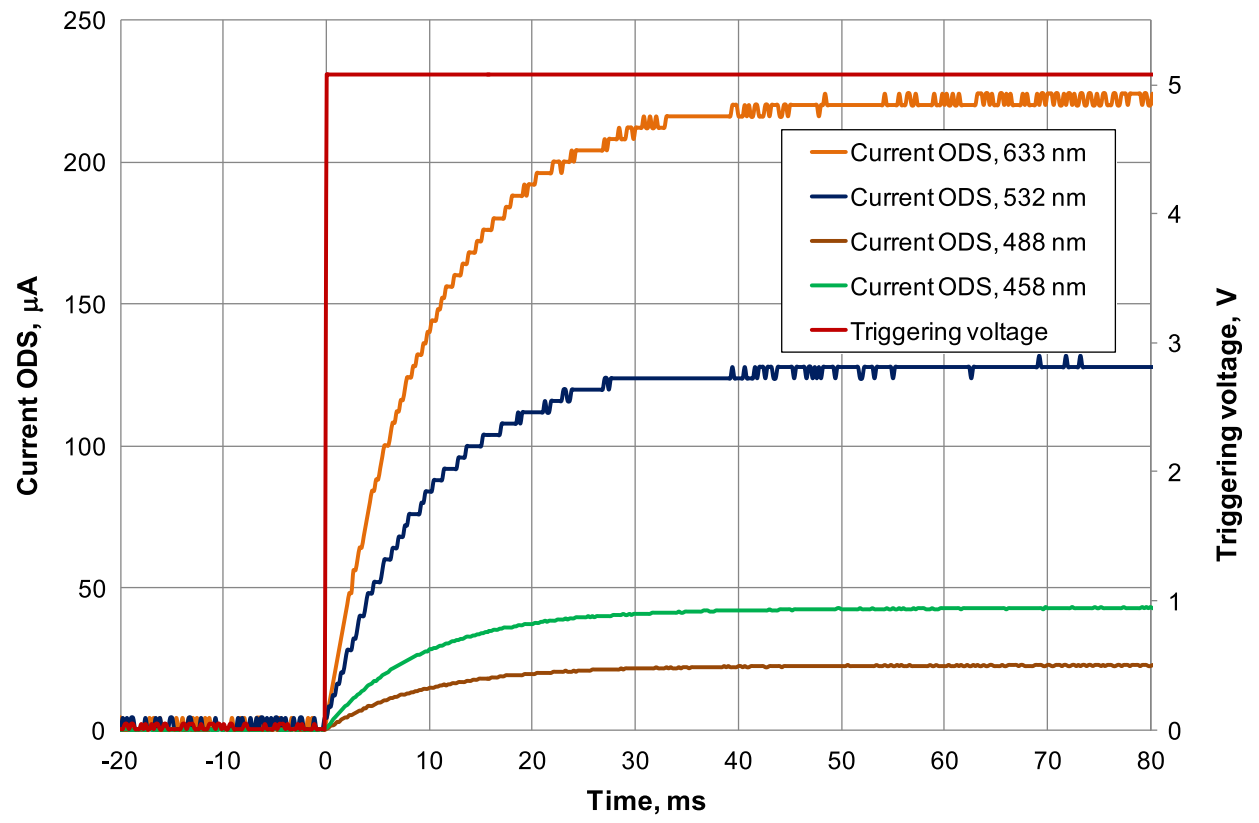

Fig. 6. Time-resolved spectrally filtered optical signal of the DC-driven CoB (ID VSL085). Triggering electrical forward voltage is shown in red for reference.

signal with respect to ambient temperature, $T_{a}$, increase is observed between 0.36 and $0.65 \%$ per ${ }^{\circ} \mathrm{C}$ depending on the filter of the ODS. The filter F488 shows the largest dependence on the temperature and the result of current measurements at different temperatures by the F488 filter of ODS is given in Figure 12.

\section{Uncertainty budget}

For the uncertainty budget evaluation we have followed the guide to the expression of measurement uncertainty (GUM) approach and the law of propagation of uncertainty (LPU) 


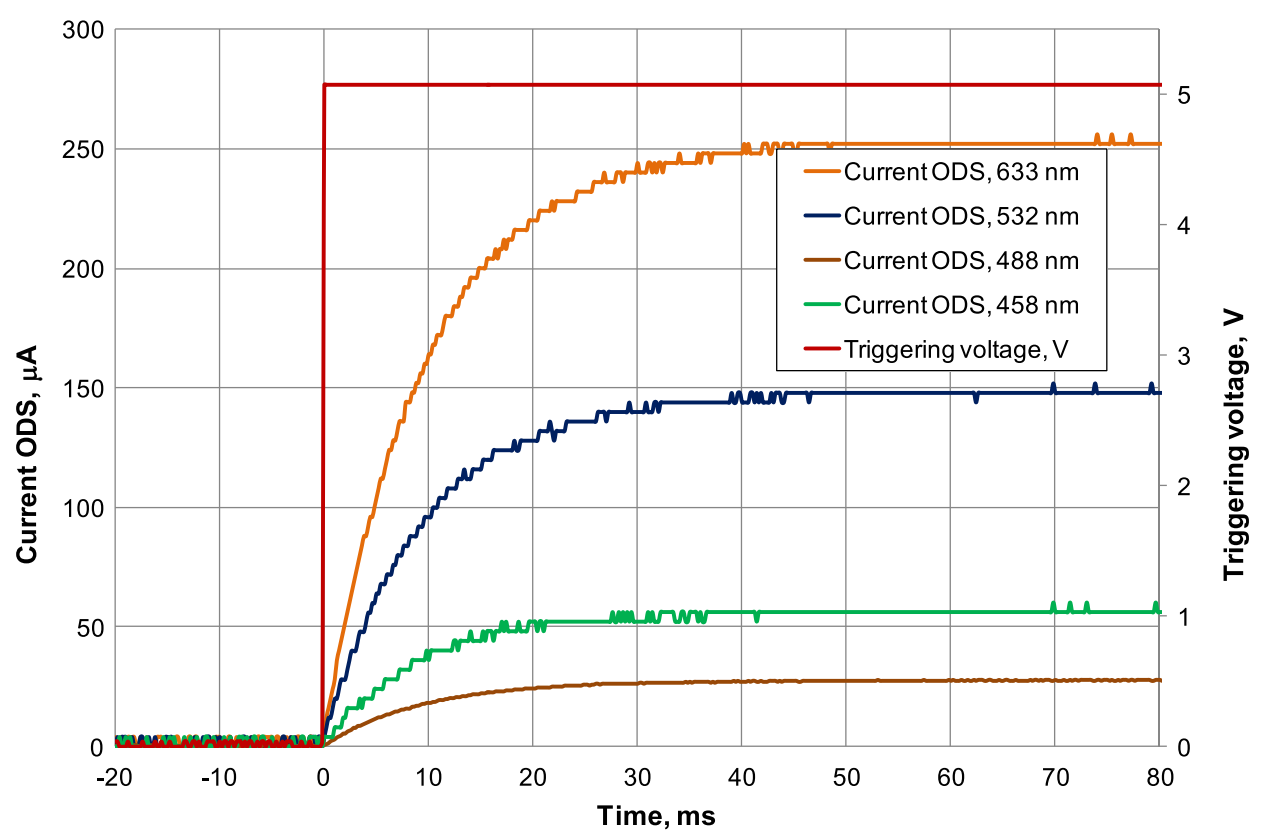

Fig. 7. Time-resolved spectrally filtered optical signal of the DC-driven CoB (ID VSL096). Triggering electrical forward voltage is shown in red for reference.

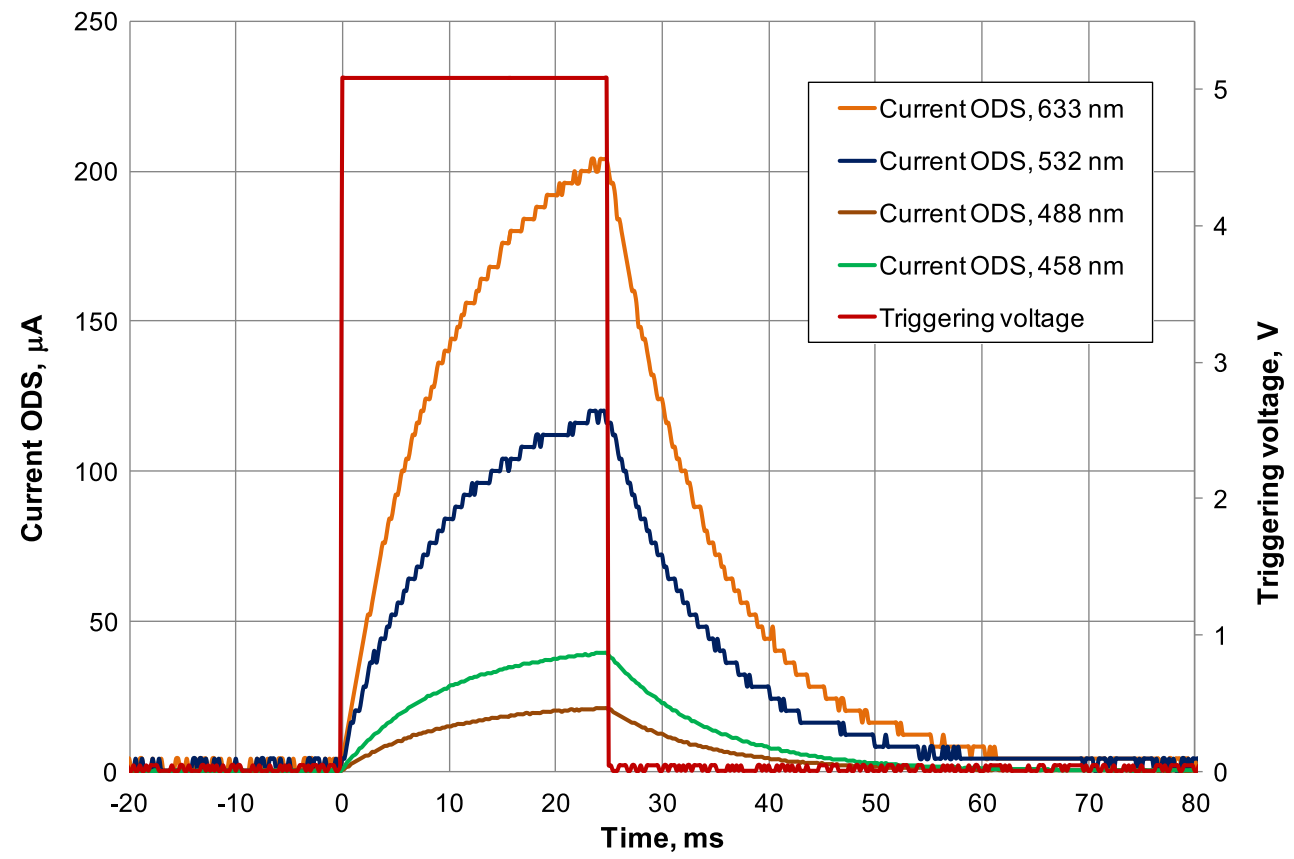

Fig. 8. Time-resolved spectrally filtered optical signal of the short-pulse driven CoB (ID VSL085). Triggering electrical forward voltage is shown in red for reference.

as it has described in [12]. The measurement uncertainty components in this study have been identified for shortpulse driven DUTs and are given in Table 8. A negligible synchronization error is expected from the variation of the width of the pulse, something below $1 / 100$ of the pulsewidth (in the range of $1 \mu \mathrm{s}$ ). The largest uncertainty component is expected from the measurement part of the high-speed short-pulse method reaching $3 \%$ due to variation of initial settings (like junction temperature of the DUTs) and the resolution of measurement instrumentation. The ambient temperature uncertainty component under pulsed operation is estimated to be at the level of $0.5 \%$. 


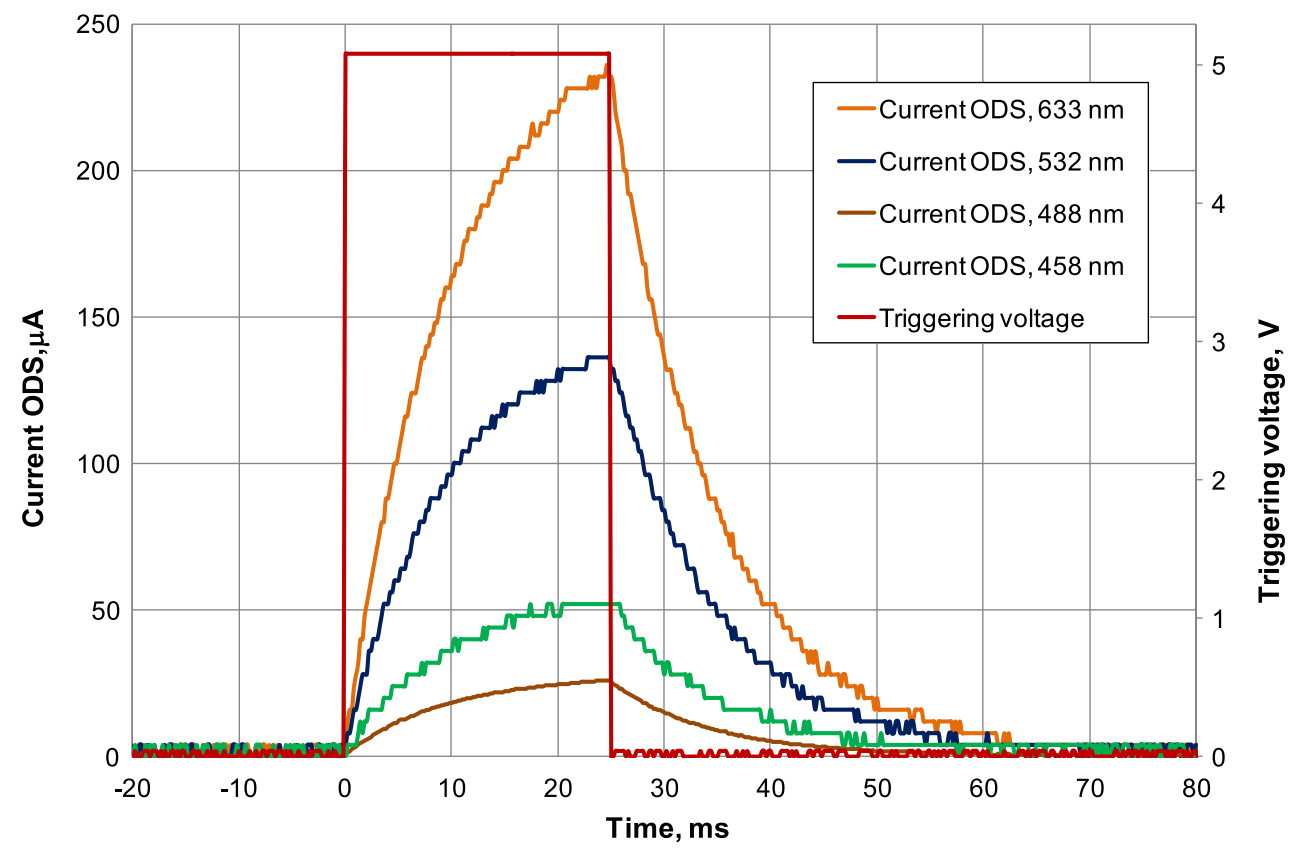

Fig. 9. Time-resolved spectrally filtered optical signal of the short-pulse driven CoB (ID VSL096). Triggering electrical forward voltage is shown in red for reference.

Table 5. Estimated centroid wavelengths of all the filters of the ODS.

\begin{tabular}{lll}
\hline Filter name, $i$ & $\lambda_{I}$ specification, nm & $\lambda_{I}$ estimated, nm \\
\hline F633, $i=1$ & 632.8 & 633.1 \\
F532, $i=2$ & 532.0 & 532.8 \\
F488, $i=3$ & 488.0 & 487.1 \\
F458, $i=4$ & 457.9 & 457.5 \\
\hline
\end{tabular}

Table 6. Estimated scaling factors $x_{i}$ for two DUTs (VSL085 and VSL096) as estimated using equation (12).

\begin{tabular}{|c|c|c|c|c|c|c|}
\hline \multirow{2}{*}{$\begin{array}{l}\text { Filter name, } \\
i\end{array}$} & \multicolumn{2}{|c|}{ Scaling factor $x_{i}$, VSL085, $\mu \mathrm{W}$} & \multirow{2}{*}{$\begin{array}{l}\text { Difference } \\
\text { pulsed \& DC } \\
\%\end{array}$} & \multicolumn{2}{|c|}{ Scaling factor $x_{i}$, VSL096, $\mu \mathrm{W}$} & \multirow{2}{*}{$\begin{array}{l}\text { Difference } \\
\text { pulsed \& DC } \\
\%\end{array}$} \\
\hline & Pulsed mode & DC mode & & Pulsed mode & DC mode & \\
\hline $\mathrm{F} 633, i=1$ & 33 & 30 & 9 & 38 & 36 & 7 \\
\hline $\mathrm{F} 532, i=2$ & 26 & 24 & 6 & 30 & 27 & 8 \\
\hline $\mathrm{F} 488, i=3$ & 29 & 27 & 9 & 34 & 31 & 8 \\
\hline $\mathrm{F} 458, i=4$ & 28 & 26 & 9 & 36 & 32 & 11 \\
\hline Average $\tilde{x}, \mu \mathrm{W}$ & 29 & 27 & 8 & 34 & 32 & 8 \\
\hline $\operatorname{STD}\left(x_{i}\right), \%$ & 10 & 9 & - & 10 & 11 & - \\
\hline
\end{tabular}

The total measurement uncertainty of the optical power of the DUT, $U\left(P_{i}\right)$, is estimated using the generalized equations (4) and (5) from [13] and the estimated individual uncertainty components from Table 8 .

\section{Discussion}

In this study, optical measurement results of two DUTs have been performed under DC and short-pulse driving. The driving condition for DC was established by setting the 


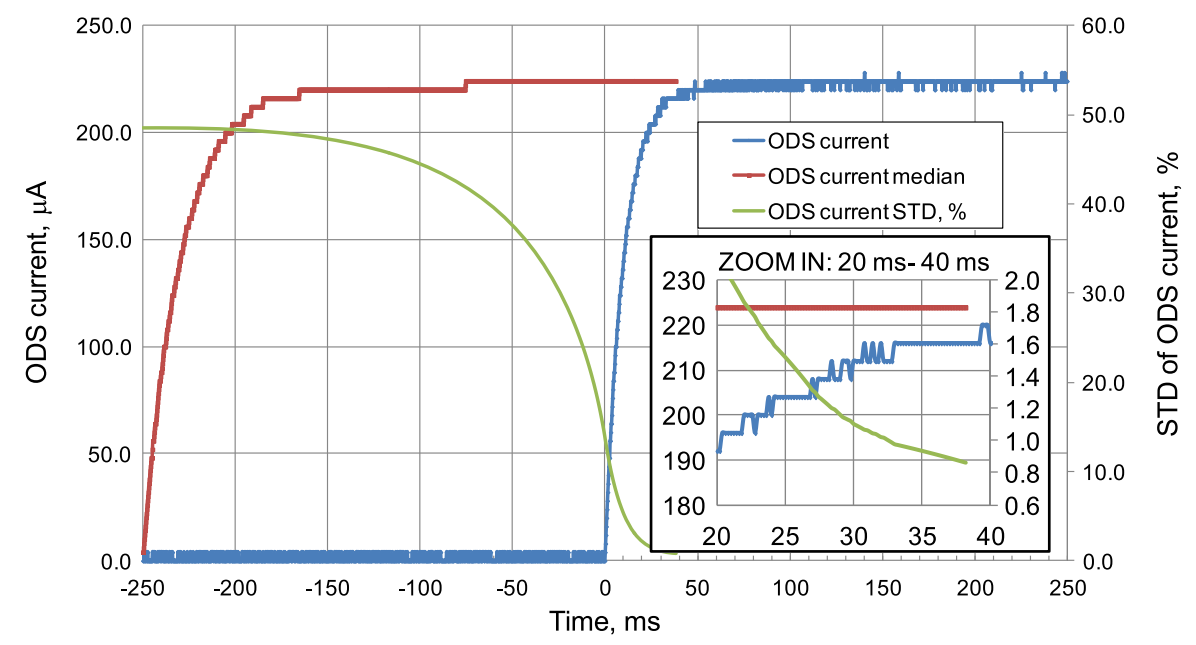

Fig. 10. Estimation of the median of the ODS current within the short pulse of width $25 \mathrm{~ms}$. The ODS measured current (filter F633, DUT VSL085) and the standard deviations of the currents used for evaluation are shown as well. The zoomed part shows the end of the iteration process at $t=38.2 \mathrm{~ms}$ : STD $=0.9 \%$ (green curve), converged current $=224 \mu \mathrm{A}$ (red curve).

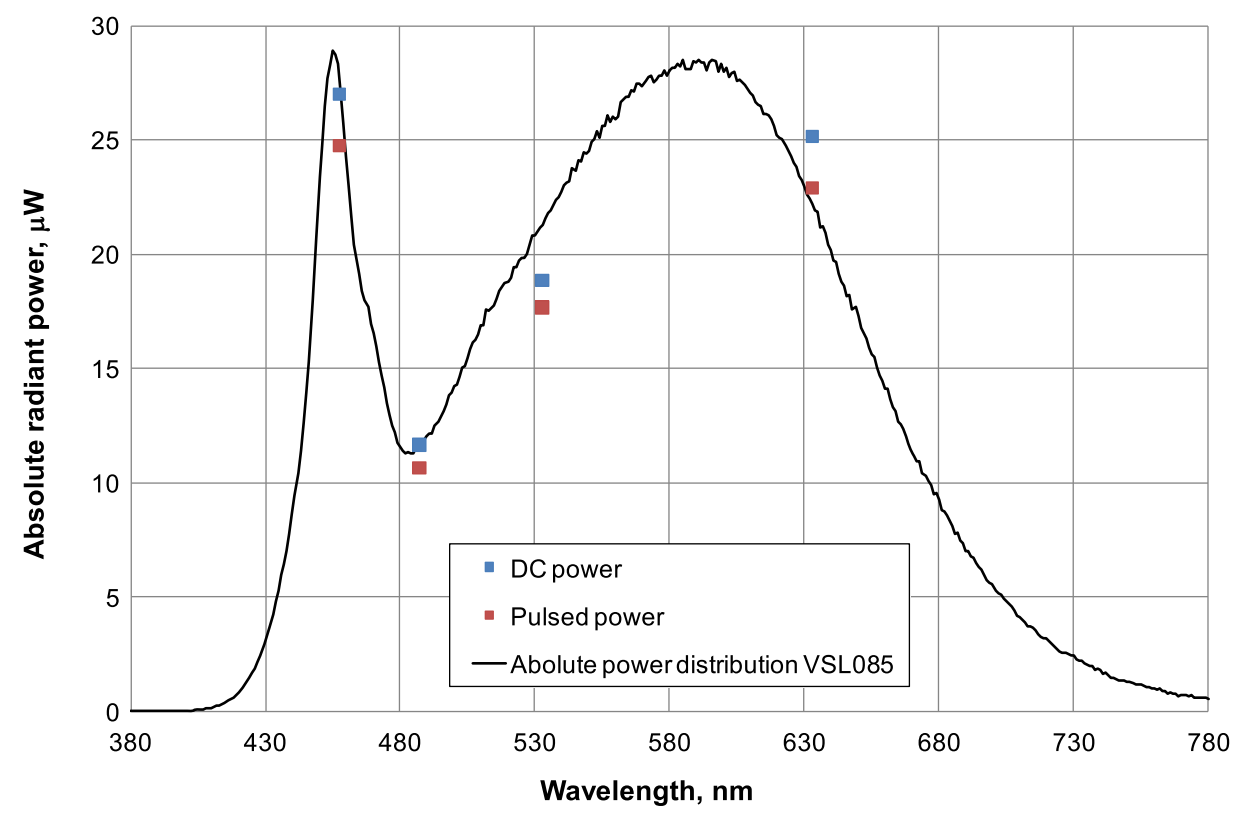

Fig. 11. Resulting absolute power of the short-pulse driven (red) and DC-driven (blue) DUT VSL085 at ODS filters wavelengths; the scaled spectral distribution function is given for the reference in black.

pulse-width to $2.5 \mathrm{~s}$ which represents a near perfect continuous DC-current, without any noticeably significant rise of the surface temperature of the DUT. This setting has been used for a comparison with short-pulse driving condition (pulse-width of $25 \mathrm{~ms}$ ).

Pulsing the DUTs with 25 ms causes a cut of the optical signal to around $8 \%$. This is clearly seen from the results given in Table 7 and shown in Figure 13.

For the tested DUTs, we advise to use a pulse-width of $40 \mathrm{~ms}$ for proper testing. The time dependency function is very much technology-dependent and should be investigated prior to the testing. We propose an iterative approach to obtain the optimal pulse-width for each technology.
Additionally, the results of the study (final traceable power) could be improved further by applying a correction to the scaling factor $x_{i}$ for the junction temperature difference. But for this, the time-resolved light output should be measured together with the time-resolved surface temperature $T_{s}$. The series of $T_{s}$ may be used to calculate the $\mathrm{p} / \mathrm{n}$ junction temperature of the DUTs as proposed in [1].

We would like to propose the method we have developed for a discussion of being suitable for LED testing in industry. Our detector-based traceability approach provides a novel insight on the testing concept. By increasing amount of spectral filters in ODS and 
Table 7. Resulting absolute power of the DC-driven and short-pulse driven DUTs VSL085 and VSL096.

\begin{tabular}{|c|c|c|c|c|c|c|c|}
\hline \multicolumn{4}{|c|}{ VSL085 } & \multicolumn{4}{|c|}{ VSL096 } \\
\hline $\begin{array}{l}\text { Centroid } \\
\text { wavelength } \\
\text { ODS filters, } \\
\text { nm }\end{array}$ & $\begin{array}{l}\text { DC power, } \\
\mu \mathrm{W}\end{array}$ & $\begin{array}{l}\text { Pulsed power, } \\
\mu \mathrm{W}\end{array}$ & $\begin{array}{l}\text { Difference, } \\
\%\end{array}$ & $\begin{array}{l}\text { Centroid } \\
\text { wavelength } \\
\text { ODS filters, } \\
\mathrm{nm}\end{array}$ & $\begin{array}{l}\text { DC power, } \\
\mu \mathrm{W}\end{array}$ & $\begin{array}{l}\text { Pulsed power, } \\
\mu \mathrm{W}\end{array}$ & $\begin{array}{l}\text { Difference, } \\
\%\end{array}$ \\
\hline 633.1 & 25 & 23 & 9 & 633.1 & 28 & 26 & 7 \\
\hline 532.8 & 19 & 18 & 6 & 532.8 & 22 & 20 & 8 \\
\hline 487.1 & 12 & 11 & 9 & 487.1 & 14 & 13 & 8 \\
\hline 457.5 & 27 & 25 & 9 & 457.5 & 35 & 31 & 11 \\
\hline
\end{tabular}

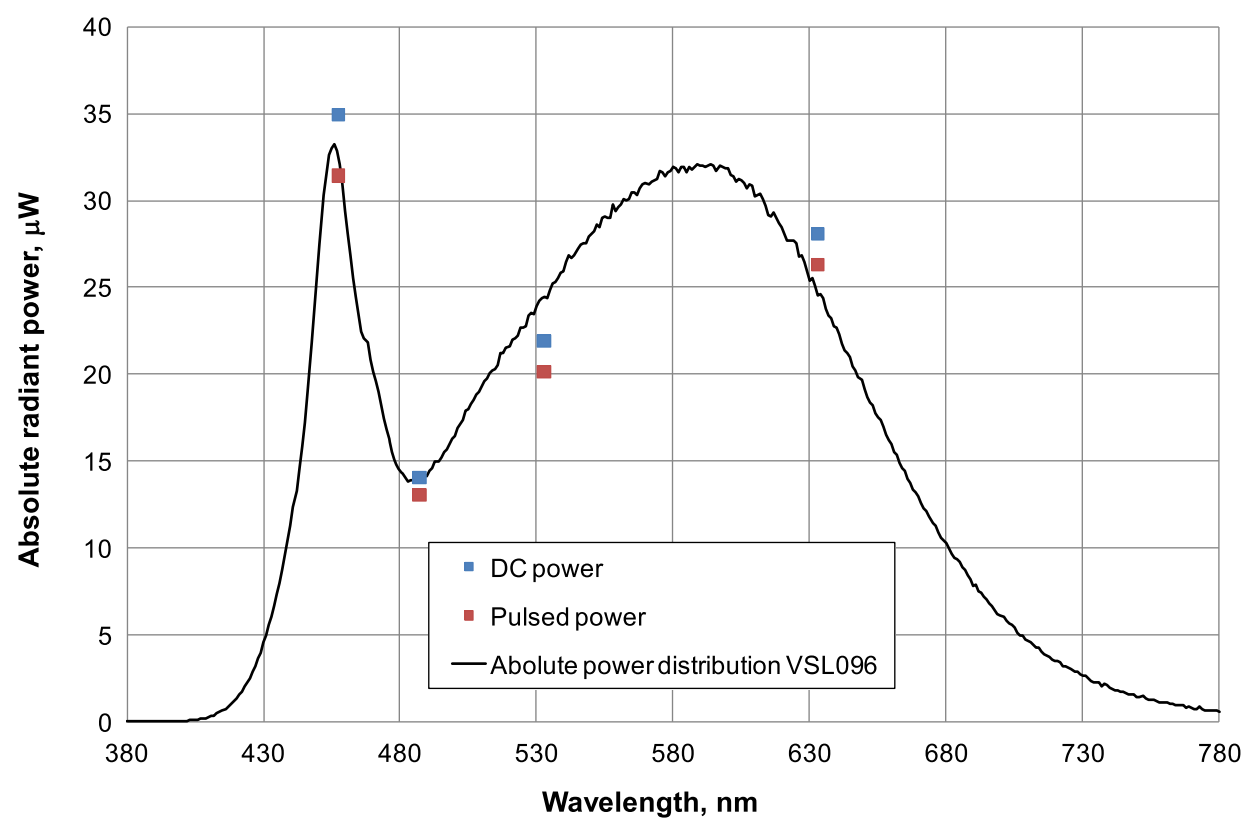

Fig. 12. Resulting absolute power of the short-pulse driven (red) and DC-driven (blue) DUT VSL096 at ODS filters wavelengths; the scaled spectral distribution function is given for the reference in black.

adding measurements of time-resolved surface temperatures, $T_{s}$, total expanded uncertainty $(k=2)$ may be reduced.

\section{Conclusions}

The short-pulse testing method used in industry to evaluate light output performance of the LEDs during production has been validated in this study. The objectives of the study were to (1) evaluate an existing testing method of the short-pulse driven DUTs, by pointing any inadequacy, (2) evaluate the major uncertainty components, and (3) to propose a high-speed testing method for traceable measurements.

When evaluating the industrial short-pulse testing method, we do conclude that the short-pulse method is a very efficient testing technique, in case a well-defined electrical current pulse is applied and the stabilization time of the device is "a priori" accurately determined. The largest inadequacy noticed is the choice of the measurand, the total luminous flux. Our conclusion is based on the fact that obtaining a traceable total luminous flux of a LED using source-based approach applicable in NMIs is not the most efficient and accurate methods of testing LEDs in industry. Transfer of a calibration factor from an incandescent standard lamp to the LED-testing industrial setup brings extra challenge due to difficulty of assuring similar testing conditions (times, temperature, driving current etc.) and misses the colour component. The largest error of the shortpulse method we notice is due to the badly-defined current pulse setting, too short (unstable signal) or too long (temperature dependency) pulse-width. Also the quality of the driving pulse may influence the quality of the output optical signal. Synchronisation issue may be pointed as an extra source of error in case signals were read out by different devices, but this issue was not investigated in this study. 
Table 8. Uncertainty budget components for short-pulse driven DUT.

\begin{tabular}{lll}
\hline Contributor & Component & $\begin{array}{l}\text { Uncertainty optical power, } \\
U\left(P_{i}\right), k=1, \%\end{array}$ \\
\hline \multirow{4}{*}{ Measurement setup } & Calibration of ODS (spectral responsivity [11]) & $1.0 \%$ \\
& System (repeatability, alignment, stray) & $1.0 \%$ \\
Total setup & Electrical measurements (level, synchronisation, resolution) & $3.0 \%$ \\
DUT & Driving pulse (stability, pulse-width, overshoot, rise time) & $0.1 \%$ \\
Total source & & $3.3 \%$ \\
& Temporal stability and ageing & $0.1 \%$ \\
Short-pulse method & Ambient temperature stability & $0.5 \%$ \\
\multirow{2}{*}{ Total method } & & $0.5 \%$ \\
Total measurement uncertainty budget & Numerical integration (width) & $0.5 \%$ \\
Total expanded uncertainty, $U\left(P_{i}\right), k=2$ & $0.2 \%$ \\
\hline
\end{tabular}

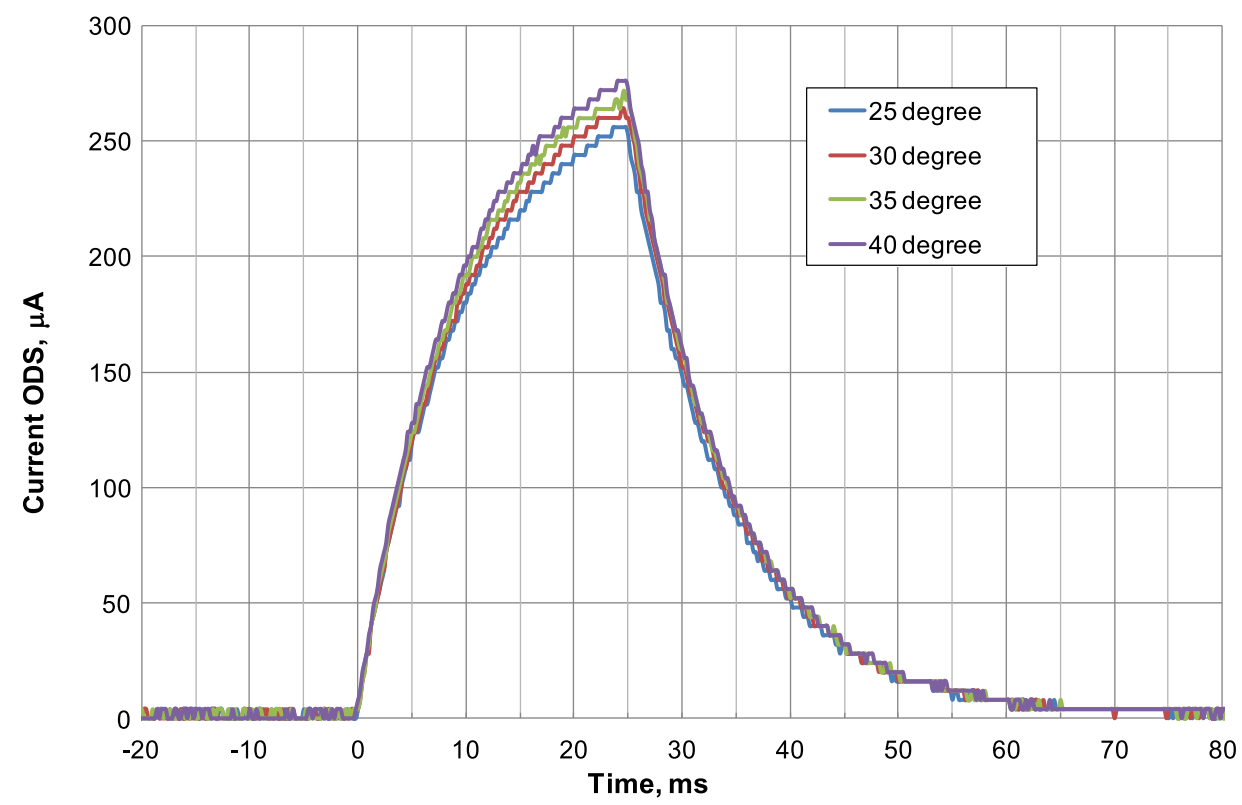

Fig. 13. Dependence of the optical output of the VSL096 from ambient temperature change when driven by a short-pulse and sensed by the filter F488 of the ODS.

We have developed a novel high-speed short-pulse detector-based traceable testing method of LEDs. Our method provides a new insight on the testing concept of LEDs: radiant power instead of luminous flux and detectorbased traceability.

We have investigated the major uncertainty components of the developed method. In industrial testing during production of LEDs the junction temperature of DUT is normally set equal to ambient temperature (which is expected to be at the range of $25 \pm 0.5^{\circ} \mathrm{C}$ ). The influence of the quality of the ambient temperature control is therefore evaluated by testing the LEDs under test at different ambient temperatures between 25 and $40{ }^{\circ} \mathrm{C}$. A rise of the amplitude of the light signal of pulse-driven DUTs is noticed, which is between 0.36 and $0.65 \% /{ }^{\circ} \mathrm{C}$, when measured by different filters of ODS. In our laboratory condition with the ambient temperature variation of $\max 0.5^{\circ} \mathrm{C}$, the deviation of lighting output is observed at the maximum level of $0.5 \%$. System component of the short-pulse measurement setup has been observed at the level of $1 \%$ which seemed to be mostly due to alignment error of the test setup, while electrical measurement component of the order of $3 \%$ was 


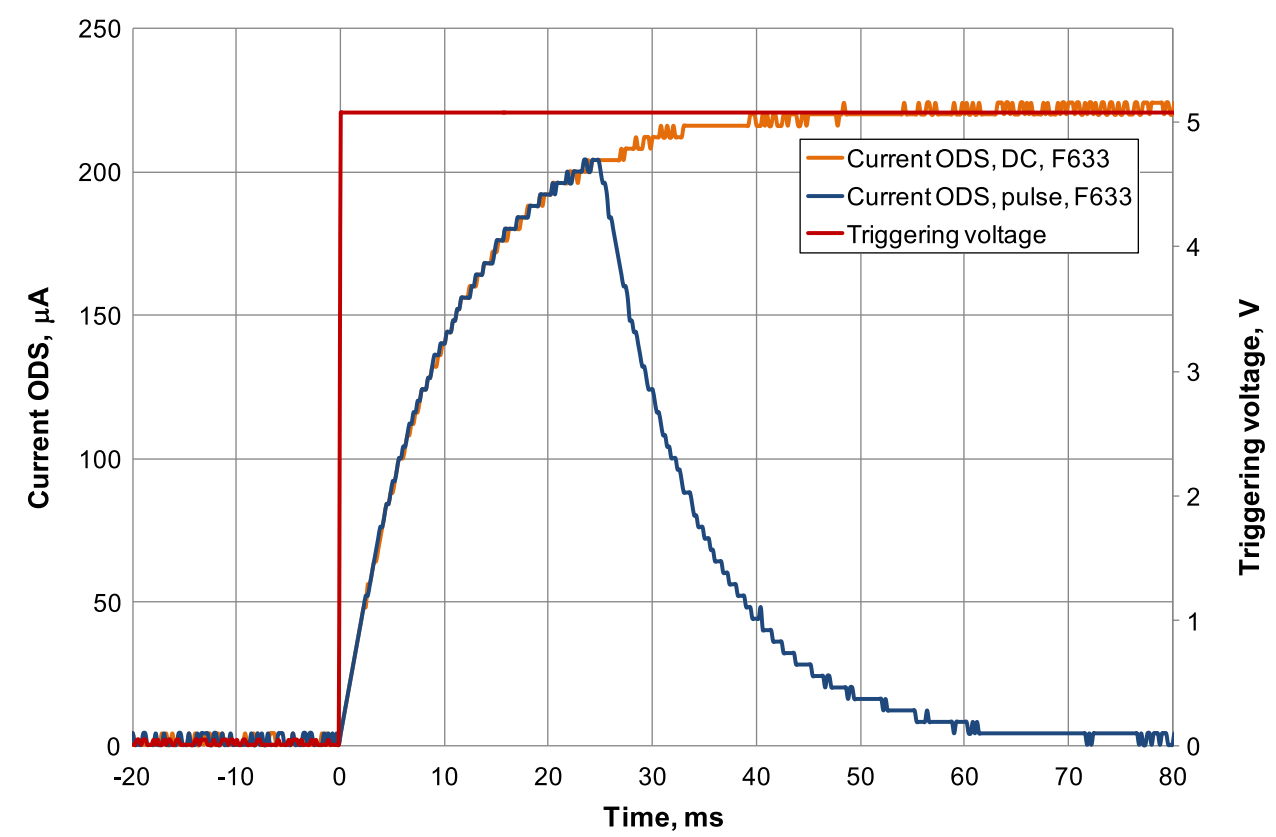

Fig. 14. Comparison of short-pulse and DC-driven resulting optical signal of DUT VSL085 as measured by the filter F633 of ODS; triggering voltage is given for reference (secondary axis).

estimated due to limitations of measurement instrument resolution, amplitude (impedance) and stability of the driving voltage.

In general, we conclude, that for the tested DUTs of type CoB in this study, the stabilisation time of an optical output for all the filters of ODS was found to be at around of $40 \mathrm{~ms}$. No spectral (colour) shift but only amplitude increase of around $8 \%$ for the whole spectrum is observed when DC measurements are substituted by the short pulse. The total expanded uncertainty of the measured total radiant power at a particular wavelength is around $U$ $\left(P_{i}\right)=7.0 \%$. By increasing amount of spectral filters in the ODS and adding measurements of time-resolved surface temperatures, $T_{s}$, the total expanded uncertainty $(k=2)$ may be significantly reduced.

\section{Implications and influences}

Industrial processes of LED production include LED light output performance testing. Most of them are monitored and controlled by optically, electrically and thermally measuring single LED and LED packages/modules by high speed short-pulse measurement methods. Development of standards regarding the LED high speed testing methods in industrial setting was initiated by the Technical Committee TC 2-64 of the Division 2 of the International Commission on Illumination (CIE) [1]. The draft report of the standard is on the final stage and it is expected to be polished within a year (personal communication), but validation will be initiated afterwards and all the contributions are of high importance.

This work shall contribute to the understanding of the traceability issues when applying the high-speed shortpulse methods for industrial testing and to help speed up the development of related standard at the European level.
Moreover, the LED manufacturing industry could benefit from this work by improving their methods, updating their instrumentation and evaluating their set-ups following recommendations and conclusion from this study.

We thank Dr. Peter Blattner (METAS), Dr. Armin Sperling (PTB) and Dr. Werner Jordan (OSRAM) for great contribution to the identification of the research objectives, the valuable discussions and feedback. This research is carried out under the EMRP project ENG62 MESaIL "Metrology for Efficient and Safe Innovative Lighting" (http://www.eng62-mesail.eu/.gr14). The EMRP is jointly funded by the EMRP participating countries within EURAMET and the European Union (http:// www.euramet.org/research-innovation/emrp/).

\section{References}

1. CIE TC2-64, High speed testing methods for LED, Draft 7.3 (2015)

2. J. Hulett, Improved photometric testing for high-power LEDs, single pulse: fast measurements, Photonics Spectra (2015)

3. LED luminaire lifetime: recommendations for testing and reporting, Solid-state lighting product quality initiative, second edn (2011)

4. CEN EN13032-4:2015, Light and lighting, Measurement and presentation of photometric data of lamps and luminaires, Part 4: LED lamps, modules and luminaires (European Committee for Standardization, 2015)

5. H.J. Kostowski, Reliable spectroradiometry (Spectroradiometry Consulting, La Plata, MD, 1997)

6. Colorimetry: understanding of the CIE system, edited by J. Schanda (Wiley-Interscience, A John-Wiey \& Sons, Inc., Hoboken, 2007) 
7. Optical radiometry, edited by A.C. Parr, U. Datla, J.L. Gardner, Vol. 41 (Elsevier Academic Press, San Diego, CA, 2005)

8. E.M. Vuelban, N. van der Leden, ENG05 Deliverable D1.1.4 report "A tristimulus meter for pulse mode operated SSL", VSL, 2013

9. L. Grodzki, The comparison of the pulse and constantcurrent LED driving, Prz. Elektrotech. (2013)
10. J. Hulett, Ensure your light measurement method doesn't undervalue your LEDs, LEDs Magazine (2017)

11. VSL instruction for spectral responsivity calibration. VSLKal-Optica/Winstr/018

12. A.B. Forbes, Approaches to evaluating measurement uncertainty, Int. J. Metrol. Qual. Eng. 3, 71-77 (2012)

13. C. Robbe, N. Nsiampa, A. Oukara, A. Papy, Quantification of the uncertainties of high-speed camera measurements, Int. J. Metrol. Qual. Eng. 5, 201 (2014)

Cite this article as: Elena Revtova, Edgar Moreno Vuelban, Dongsheng Zhao, Jacques Brenkman, Henk Ulden, Traceability validation of a high speed short-pulse testing method used in LED production, Int. J. Metrol. Qual. Eng. 8, 30 (2017) 